\title{
The small dispersion of the mid IR - hard X-ray correlation in active galactic nuclei ${ }^{\star}$
}

\author{
H. Horst ${ }^{1,2}$, A. Smette ${ }^{1}$, P. Gandhi ${ }^{3,1}$, and W. J. Duschl ${ }^{2,4}$ \\ 1 European Southern Observatory, Casilla 19001, Santiago 19, Chile \\ e-mail: hhorst@eso.org \\ 2 Zentrum für Astronomie, ITA, Universität Heidelberg, Albert-Ueberle-Str. 2, 69120 Heidelberg, Germany \\ 3 Institute of Astronomy, Madingley Road, Cambridge CB3 OHA, UK \\ 4 Steward Observatory, The University of Arizona, 933 N. Cherry Ave, Tucson, AZ 85721, USA
}

Received 13 June 2006 / Accepted 27 July 2006

\section{ABSTRACT}

\begin{abstract}
Context. We investigate mid-infrared and X-ray properties of the dusty torus in unification scenarios for active galactic nuclei. Aims. We use the relation between mid IR and hard X-ray luminosities to constrain AGN unification scenarios.

Methods. With VISIR at the VLT, we have obtained the currently highest angular resolution $(0$ ' $35 F W H M)$ narrow-band mid infrared images of the nuclei of 8 nearby Seyfert galaxies. Combining these observations with X-ray data from the literature we study the correlation between their mid IR and hard X-ray luminosities.

Results. We find that the rest frame $12.3 \mu \mathrm{m}\left(L_{\mathrm{MIR}}\right)$ and 2-10 $\mathrm{keV}\left(L_{\mathrm{X}}\right)$ luminosities are correlated at a highly significant level. The best fit power-law to our data is $\log L_{\mathrm{MIR}} \propto(1.60 \pm 0.22) \log L_{\mathrm{X}}$, showing a much smaller dispersion than earlier studies.

Conclusions. The similarity in the $\log L_{\mathrm{MIR}} / \log L_{\mathrm{X}}$ ratio between Sy1s and Sy2s even using high angular resolution MIR data implies that the similarity is intrinsic to AGN and not caused by contamination from extra-nuclear emission. This supports clumpy torus models. The exponent of the correlation constrains the inner geometry of the torus.
\end{abstract}

Key words. galaxies: active - infrared: galaxies $-\mathrm{X}$-rays: galaxies

\section{Introduction}

The unification model for active galactic nuclei (AGN) interprets the different appearance of Seyfert 1 (Sy1) and Seyfert 2 (Sy2) galaxies uniquely as the result of an orientation effect (Antonucci 1993; Barthel 1994; Urry \& Padovani 1996). The central engine is considered to be surrounded by an optically and geometrically thick molecular torus. Associated with this torus are large masses of dust that supposedly reprocess the X-ray and UV emission from the accretion disk and re-emit it in the mid infrared (MIR) regime (Pier \& Krolik 1993).

It is thus very attractive to search for correlations between IR continuum and hard X-ray emission in order to test the unification scenarios for AGN. A tight correlation between the $10.5 \mu \mathrm{m}$ continuum and the absorption-corrected 2-10 keV luminosities for 8 nearby Seyfert galaxies was reported by Krabbe et al. (2001) using 1".2 resolution MIR data. More recently Lutz et al. (2004) found a correlation between the rest frame $6 \mu \mathrm{m}$ luminosity and the absorption-corrected hard X-ray luminosity for a sample of 71 AGN. This sample was comprised of objects for which 24" angular resolution ISOPHOT spectra and hard $\mathrm{X}$-ray observations were available; in particular it does not contain Compton-thick objects. However, the authors reported two problems that the unification model faces when compared with their observations. (I) The scatter of the relation is about an order of magnitude larger than expected from the results of Krabbe et al. (2001). (II) There is no significant difference between early type (Sy types 1 to 1.5 ) and late type (Sy types 1.8 to 2) objects in the average ratio of mid-infrared to hard X-ray emission,

\footnotetext{
* Based on ESO observing programme 075.B-0844(C).
}

as would be expected from an optically and geometrically thick torus dominating the mid-IR AGN continuum.

Here we study this relation through rest wavelength $12.3 \mu \mathrm{m}$ photometry of AGN, improving upon Krabbe et al. (2001) and Lutz et al. (2004) by a factor of 3 and 80, respectively, in terms of angular resolution. The wavelength was chosen as, among the bands accessible from the ground, it best traces hot and warm dust.

Throughout this paper we assume $H_{0}=72 \mathrm{~km} \mathrm{~s}^{-1}, \Omega_{\Lambda}=$ 0.73 and $\Omega_{\mathrm{m}}=0.27$ (Spergel et al. 2003).

\section{Observations and data analysis}

Between March and July 2005 we observed 8 nearby Seyfert nuclei (see Table 1), selected from the sample used by Lutz et al. (2004), with VISIR (Lagage et al. 2004) at the VLT. The individual objects were selected as to avoid a strong bias toward higher luminosities with higher redshift. All observations were carried out under good photometric and optical seeing conditions $\left(\leq 0 .{ }^{\prime} 8\right)$ and with airmass below 1.3. For all observations we used the parallel chop/nod mode of VISIR with a chopping throw of $8^{\prime \prime}$, thus receiving all three beams on the detector. We observed each object in three different narrow-band filters in order to obtain the rough shape of its MIR spectral energy distribution (SED). These filters were chosen as to avoid emission and absorption lines, in particular the $12.8 \mu \mathrm{m}$ NeII line and the broad $9.7 \mu \mathrm{m}$ silicate feature which in cases of very strong absorption can extend to $\lambda \approx 12.5 \mu \mathrm{m}$ (Dorschner et al. 1978). NGC 7314 was only observed in two different filters. Total integration times ranged between 1.2 and 9.2 min per filter and per object. 
Table 1. Basic parameters of our sample of galaxies. Heliocentric redshifts $z$ were taken from the NED, Seyfert types are according to Véron-Cetty \& Véron (2000). The absorption corrected hard X-ray luminosities we compiled from the literature. Where multiple observations were available the average was taken. In these cases the reported uncertainties correspond to $1 / 2$ of the peak-to-peak variabilities. In the case of PG $2130+099$ only one measurement could be found. Here we assume a variability of a factor of 2 . The last column contains the physical scales resolved by VISIR, computed for an angular resolution of $0 ! 35$, which is a typical value for our observations (see Table 2).

\begin{tabular}{lcclc}
\hline \hline Object & $z$ & Sy & $\log L_{2-10 \mathrm{keV}}$ & Scale [pc] \\
\hline Fairall 9 & 0.047 & 1.2 & $43.84 \pm 0.15^{a, b, c}$ & 320 \\
NGC 526a & 0.019 & 1.9 & $43.14 \pm 0.35^{a, c, k, l}$ & 135 \\
NGC 3783 & 0.010 & 1.5 & $43.12 \pm 0.15^{a, c, d}$ & 70 \\
NGC 4579 & 0.005 & $1.9^{*}$ & $41.18 \pm 0.22^{c, e, f, i}$ & 35 \\
NGC 4593 & 0.009 & 1.0 & $42.89 \pm 0.15^{a, c, g, h}$ & 65 \\
PKS 2048-57 & 0.011 & 2.0 & $43.07 \pm 0.15^{c, o}$ & 80 \\
PG 2130+099 & 0.062 & 1.5 & $43.68 \pm 0.15^{c, n}$ & 415 \\
NGC 7314 & 0.005 & 1.9 & $42.19 \pm 0.15^{a, c, k, m}$ & 35 \\
\hline
\end{tabular}

References are ${ }^{a}$ Turner \& Pounds (1989), ${ }^{b}$ Gondoin et al. (2001), ${ }^{c}$ Lutz et al. (2004), ${ }^{d}$ Reeves et al. (2004), ${ }^{e}$ Terashima et al. (2002), ${ }^{f}$ Ho et al. (2001), ${ }^{g}$ Reynolds et al. (2004), ${ }^{h}$ Steenbrugge et al. (2003), ${ }^{i}$ Cappi et al. (2005), ${ }^{k}$ Risaliti (2002), ${ }^{l}$ Landi et al. (2001), ${ }^{m}$ Kraemer et al. (2004), ${ }^{n}$ Risaliti et al. (2000), ${ }^{o}$ Smith \& Done (1996).

${ }^{*}$ For a discussion of the classification of NGC 4579 see Sect. 3.

We reduced science and standard star frames using the pipeline written by Eric Pantin (private communication). To eliminate glitches, the pipeline applies a bad pixel mask and removes detector stripes. Subsequently we removed background variations using a 2 dimensional polynomial fit. Here we treated each nodding cycle separately as the background pattern sometimes changes between two consecutive cycles. The count rate for one full exposure was calculated as the mean of all 3 beams from all nodding cycles of this exposure. As an error estimate we use the standard deviation of these. In order to minimise the effect of residual sky background we chose relatively small apertures $\left(\approx 10\right.$ pixels $\left.=11^{\prime \prime} 27\right)$ for the photometry and corrected the obtained count rates using the radial profiles of standard stars. Finally we calibrated our photometry using the same standard stars. These were observed within $1.5 \mathrm{hrs}$ of the science exposures and with a maximum airmass difference of 0.15 . Our observational results are shown in Table 2. Comparing the FWHM of standard star and science exposures yields no indication that our sources are extended. Thus our observations indeed probe the physical scales given in Table 1.

\section{Results}

Figure 1 shows all photometric data points taken during our observing programme. For comparison Fig. 2 shows our measurements together with ISOPHOT spectra taken from Rigopoulou et al. (1999) for these three sources common to both samples. Among our data the SED of PKS 2048-57 is the only one displaying a strong $9.7 \mu \mathrm{m}$ silicate feature which, however, does not significantly affect our measurement of the continuum flux in the NeIIref1 filter. This is important as it validates our result on the MIR - hard X-ray correlation (see below).

One of the objects in our sample - NGC 4579 - has peculiar MIR properties. Comparing the ISOPHOT $6 \mu \mathrm{m}$ flux reported in Lutz et al. (2004) of $S_{6 \mu \mathrm{m}}=96 \mathrm{mJy}$ to our VISIR flux levels (see Table 2) leads to the conclusion that either this object has a peculiar MIR SED that does not rise toward longer wavelengths

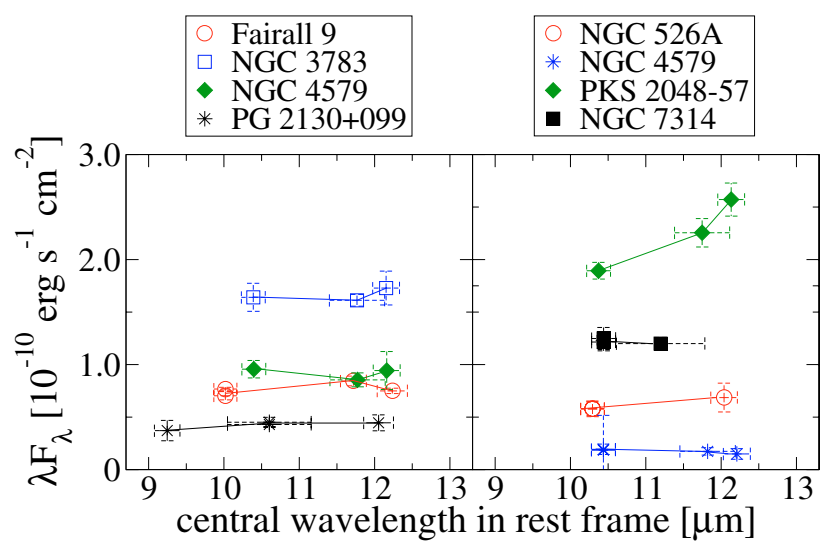

Fig. 1. The MIR SEDs of our sample of galaxies. Early- and latetype Seyferts are shown in the left- and right-hand panel respectively. Horizontal error bars show the band width of the used filters. The flux densities of NGC 7314 have been offset by $+1 \times 10^{-10} \mathrm{erg} \mathrm{s}^{-1} \mathrm{~cm}^{-2}$ for clarity.

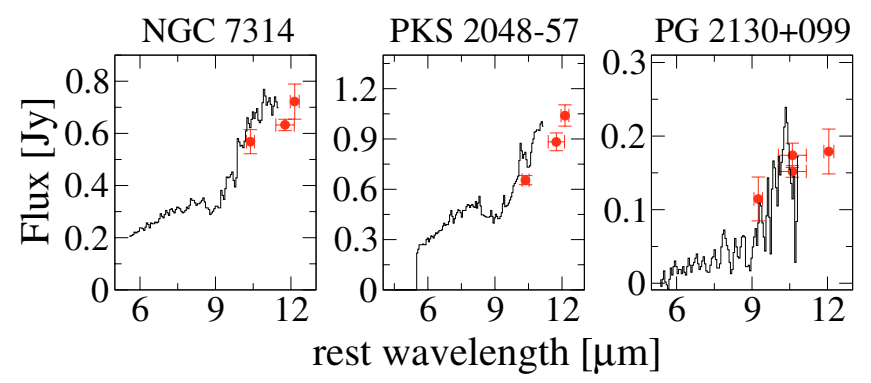

Fig. 2. Comparison of ISOPHOT-S spectra (dashed histograms) taken from Rigopoulou et al. (1999) and our VISIR photometry (filled circles). Horizontal error bars show the band width of the filters used.

as expected for typical AGN or that the bulk of IR emission $(\gtrsim 70 \%)$ does not originate in the central point source. In both cases it is highly questionable whether the latter is dominated by a molecular torus. Moreover Contini (2004) reports the MIR emission of NGC 4579 to be dominated by thermal emission of shock-heated ISM in the vicinity of the nuclear region rather than by reprocessed X-ray and UV emission from the central engine itself. These peculiarities plus the fact that NGC 4579 has also been classified as a low-ionisation nuclear emission region (LINER) by Keel (1983) and Filippenko \& Sargent (1985) led us to exclude it from the MIR - hard X-ray correlation investigated here. NGC 7314, the other low luminosity object in our sample, harbours a regular Seyfert nucleus (Lumsden et al. 2004).

For all objects in our sample we calculated the $12.3 \mu \mathrm{m}$ rest frame luminosity by using the filter with the central wavelength closest to $12.3 \mu \mathrm{m}$ and assuming a flat SED in this spectral region. Then we compiled absorption-corrected 2-10 keV measurements for our sample from the literature (see Table 1 for details). When several such measurements were available we use the mean luminosity for the MIR - hard X-ray correlation and take half of the peak-to-peak-variability as a conservative estimate of the uncertainty in the hard X-ray luminosity. Only one such measurement could be found for PG 2130+099 for which we therefore assumed a variability of a factor of 2 . As we derive the luminosity distances of all targets solely from their redshifts we assume an uncertainty of $3 \mathrm{Mpc}$ due to possible deviations from the Hubble flow. This is applied to the error bars of both $\mathrm{X}$-ray and MIR luminosities. 
Table 2. Basic observational parameters for all data used: Object name, observing date (in 2005), filter, flux and full width half maximum for standard star (STD) and science observations. As error estimates we use the standard deviation of all contributing beams (see section 2 for details). The FWHM are the average of all beams of one exposure. All science and corresponding STD exposures were obtained within a maximum airmass difference of 0.15 .

\begin{tabular}{|c|c|c|c|c|c|c|c|c|c|c|c|}
\hline \multirow[t]{2}{*}{$\overline{\overline{\text { Object }}}$} & \multirow{2}{*}{$\begin{array}{l}\text { Obs. date } \\
\text { (MM-DD) }\end{array}$} & \multirow[t]{2}{*}{ Filter* } & \multirow[t]{2}{*}{ Flux [mJy] } & \multicolumn{2}{|c|}{$F W H M\left[{ }^{\prime \prime}\right]$} & \multirow[t]{2}{*}{ Object } & \multirow{2}{*}{$\begin{array}{l}\text { Obs. date } \\
\text { (MM-DD) }\end{array}$} & \multirow[t]{2}{*}{ Filter* } & \multirow[t]{2}{*}{ 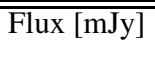 } & \multicolumn{2}{|c|}{$F W H M\left[{ }^{\prime \prime}\right]$} \\
\hline & & & & STD & Obj & & & & & STD & Obj \\
\hline Fairall 9 & $07-21$ & SIV & $256 \pm 5$ & 0.32 & 0.35 & NGC 4593 & $04-30$ & SIV & $331 \pm 29$ & 0.30 & 0.31 \\
\hline Fairall 9 & $07-21$ & NeIIref1 & $330 \pm 18$ & 0.38 & 0.37 & NGC 4593 & $04-30$ & PAH2ref2 & $335 \pm 26$ & 0.33 & 0.33 \\
\hline Fairall 9 & $07-21$ & NeII & $306 \pm 10$ & 0.40 & 0.37 & NGC 4593 & $04-30$ & NeIIref1 & $382 \pm 73$ & 0.34 & 0.36 \\
\hline Fairall 9 & $07-21$ & SIV & $235 \pm 13$ & 0.32 & 0.34 & PKS 2048-57 & $06-10$ & PAH2ref2 & $883 \pm 53$ & 0.38 & 0.40 \\
\hline NGC 526a & $07-20$ & SIV & $199 \pm 26$ & 0.41 & 0.30 & PKS 2048-57 & $06-10$ & NeIIref1 & $1040 \pm 64$ & 0.41 & 0.43 \\
\hline NGC 526a & $07-20$ & NeIIref1 & $275 \pm 55$ & 0.41 & 0.35 & PKS 2048-57 & $06-10$ & SIV & $655 \pm 28$ & 0.37 & 0.39 \\
\hline NGC 526a & $07-20$ & SIV & $199 \pm 22$ & 0.41 & 0.32 & PG 2130+099 & $06-10$ & РAH2 & $152 \pm 8$ & 0.39 & 0.38 \\
\hline NGC 3783 & 04-17 & SIV & $568 \pm 46$ & 0.29 & 0.31 & PG 2130+099 & $06-10$ & SIVref1 & $115 \pm 20$ & 0.35 & 0.36 \\
\hline NGC 3783 & $04-17$ & PAH2ref2 & $632 \pm 22$ & 0.34 & 0.35 & PG 2130+099 & $06-10$ & NeII & $179 \pm 31$ & 0.41 & 0.43 \\
\hline NGC 3783 & 04-17 & NeIIref1 & $722 \pm 67$ & 0.34 & 0.36 & PG 2130+099 & $06-10$ & PAH2 & $174 \pm 17$ & 0.39 & 0.40 \\
\hline NGC 4579 & $04-30$ & SIV & $67 \pm 17$ & 0.30 & 0.25 & NGC 7314 & $07-20$ & SIV & $75 \pm 29$ & 0.40 & 0.36 \\
\hline NGC 4579 & $04-30$ & PAH2ref2 & $69 \pm 14$ & 0.33 & 0.29 & NGC 7314 & $07-20$ & PAH2 & $75 \pm 22$ & 0.36 & 0.37 \\
\hline NGC 4579 & $04-30$ & NeIIref1 & $61 \pm 21$ & 0.34 & 0.30 & NGC 7314 & $07-20$ & SIV & $87 \pm 37$ & 0.40 & 0.34 \\
\hline NGC 4579 & 04-30 & SIV & $64 \pm 12$ & 0.30 & 0.32 & & & & & & \\
\hline
\end{tabular}

* The central wavelengths for the individual filters are $9.82 \mu \mathrm{m}$ for SIVref1, $10.49 \mu \mathrm{m}$ for SIV, $11.25 \mu \mathrm{m}$ for PAH, $11.88 \mu \mathrm{m}$ for PAH2ref2, $12.27 \mu \mathrm{m}$ for NeIIref1 and $12.81 \mu \mathrm{m}$ for NeII. Their transmission curves are shown in the VISIR User's manual at http://www.eso.org/instruments/visir/

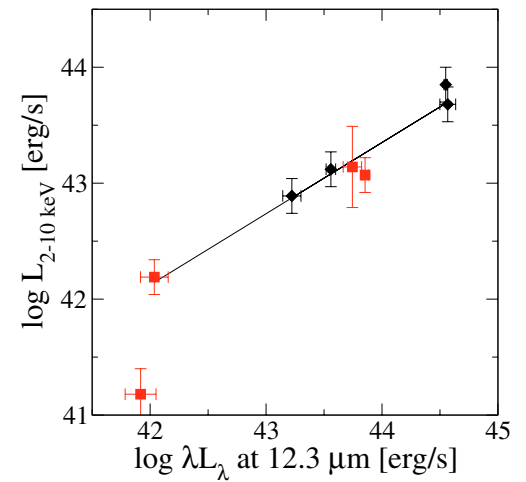

Fig. 3. Correlation of MIR to absorption-corrected hard X-ray luminosities for our VISIR sample. Filled diamonds are early-type Seyferts (Type Sy 1.5 or smaller) while filled squares are late-type Seyferts. The power law displayed is the best fit obtained when omitting NGC 4579 (see Sect. 3 for details).

The resulting MIR - hard X-ray correlation we obtain for our VISIR sample is shown in Fig. 3. Pearson's coefficient is $r=0.98$ meaning that the correlation is highly significant. The best fit power-law to our data is $\log L_{\mathrm{MIR}}=(-25.4 \pm 9.5)+$ $(1.60 \pm 0.22) \cdot \log L_{X}$ with measurement errors considered in the fit. Including NGC 4579 into the fit yields an exponent of $\sim 1.28$ rather than 1.6 and $r=0.95$.

\section{Discussion and conclusions}

We have presented new high-resolution MIR observations of 8 nearby Seyferts, obtained with VISIR at the VLT. We found that their rest-frame $12.3 \mu \mathrm{m}$ and absorption-corrected 2-10 keV luminosities are strongly correlated following $L_{\mathrm{MIR}} \propto L_{\mathrm{X}}^{1.6}$. Earlyand late-type Seyfert galaxies follow the same relation. At the low-luminosity end the correlation is not well defined as one of the two object's MIR radiation is very probably not dominated by a dusty torus.
Both Krabbe et al. (2001) and Lutz et al. (2004) found a correlation close to $L_{\mathrm{MIR}} \propto L_{\mathrm{X}}^{1}$. The exponent different to the one we find is most probably due to the difference in angular resolution (24" for ISOPHOT, 1'. 2 for Krabbe et al.'s sample and $\sim 0$.'35 for VISIR) and to the choice of the rest frame wavelength as the intervening medium is expected to be more optically thin at $12.3 \mu \mathrm{m}$ than at shorter wavelengths. In particular, using the corresponding ISOPHOT $6 \mu \mathrm{m}$ data for our sample also yields an exponent close to 1 . In addition we find a dispersion similar to the one in Krabbe et al. (2001); the larger one reported by Lutz et al. (2004) can be attributed to their relatively poor angular resolution and the fact that the emission at $6 \mu \mathrm{m}$ is slightly more dependent on inclination effects (e.g. see Hönig et al. 2006). On the other hand we confirm the absence of a significant difference in the $L_{\mathrm{MIR}} / L_{\mathrm{X}}$ ratio between early and late-type objects found by both other studies. At our high angular resolution this is clearly in disagreement with smooth torus models, in which a flux difference of one order of magnitude is expected for type 1 and type 2 AGN of the same bolometric luminosity (e.g. Pier \& Krolik 1993). This cannot be accounted for by dust clumps situated in the ionisation cone (Efstathiou et al. 1995) because their contribution to the nuclear MIR flux should not exceed 50\% (see Galliano et al. 2005). Clumpy torus models (e.g. Nenkova et al. 2002) do not predict such an offset and seem to be compatible with our results.

Hönig et al. (2006) recently used 3D radiative transfer modelling to demonstrate that clumpy tori can appear as optically thin in the MIR with most of the radiation originating in the innermost part of the torus. In their model individual clouds are optically thick but their filling factor is very small. Consequently lines-of-sight toward the innermost clouds are usually not blocked by other clouds. This implies that $L_{\mathrm{MIR}}$ is proportional to the surface area $A$ of the inner edge of the torus and the covering factor of clouds projected on $A$. Our finding that Sy1s and Sy2s follow the same $L_{\mathrm{X}}-L_{\mathrm{MIR}}$ relation is a strong indication that the torus appears optically thin at $12.3 \mu \mathrm{m}$.

The correlation we find then requires a dependency of $A$ or the covering factor on $L_{\mathrm{X}}$ and thus the bolometric luminosity $L_{\mathrm{Bol}}$. The simplest explanation for this observed effect is 
flaring: If the torus' inner edge is defined by the dust sublimation radius $r_{0}$, we obtain $A=2 \pi r_{0} \cdot H\left(r_{0}\right)$ where $H(r)$ is the scale height of the cloud distribution. For a flaring disk $H(r)$ can be written as $H(r) \propto r^{\alpha}$ with $\alpha$ being constant. This assumption yields $A \propto r_{0}^{1+\alpha}$. The dust sublimation radius $r_{0}$ follows $r_{0} \propto L_{\mathrm{Bol}}^{0.5} \propto L_{\mathrm{X}}^{0.5}$. This leads to $L_{\mathrm{MIR}} \propto L_{\mathrm{X}}^{0.5+0.5 \alpha}$. Our observations yield $L_{\mathrm{MIR}} \propto L_{X}^{1.6}$ and thus $\alpha \approx 2$. Including NGC 4579 results in $\alpha \approx 1.5$.

Another possibility is that its scale height depends on the radial accretion rate $\dot{M}$. The torus model presented by Beckert \& Duschl (2004) predicts $H(r) / r \propto \dot{M}^{0.5}$. For AGN with comparable radiative efficiencies $\dot{M}$ is proportional to $L_{\mathrm{X}}$. This yields $L_{\mathrm{MIR}} \propto A \propto L_{\mathrm{X}}^{1.5}$ which is very close to the correlation we find.

The high angular resolution of our MIR observations indicates that the similarity of the $L_{\mathrm{MIR}} / L_{\mathrm{X}}$ ratio for Sy1s and Sy2s is intrinsic rather than caused by extra-nuclear emission. Although contributions of dust clumps situated in the ionisation cone cannot be ruled out they should not dominate the nuclear MIR flux. Assuming our sample is representative of the general AGN population our results thus support recent models of clumpy dust tori (e.g. Nenkova et al. 2002; Hönig et al. 2006) and, furthermore, indicate that the filling factor of clouds is small. The geometry of the torus can be constrained by the slope of the $L_{\mathrm{X}}-L_{\mathrm{MIR}}$ correlation. Clumpy tori with low filling factors might also account for the similarity of Spitzer spectra of type 1 and type 2 QSOs (Sturm et al. 2006).

Acknowledgements. We thank Thomas Beckert, Sebastian Hönig and Eric Pantin for helpful discussions and an anonymous referee for comments and suggestions. This research made use of the NASA/IPAC Extragalactic Database (NED) which is operated by the Jet Propulsion Laboratory, California Institute of Technology, under contract with the National Aeronautics and Space Administration.

\section{References}

Antonucci, R. 1993, ARA\&A, 31, 473

Barthel, P. D. 1994, in The First Stromlo Symposium: The Physics of Active Galaxies, ed. G. V. Bicknell, M. Dopita, \& P. Quinn, 54, 175

Beckert, T., \& Duschl, W. J. 2004, A\&A, 426, 445

Cappi, M., Panessa, F., Bassani, L., et al. 2005, [arXiv:astro-ph/0509584]

Contini, M. 2004, MNRAS, 454, 675

Dorschner, J., Friedemann, C., \& Gürtler, J. 1978, Ap\&SS, 54, 181

Efstathiou, A., Hough, J. H., \& Young, S. 1995, MNRAS, 277, 1134

Filippenko, A. V., \& Sargent, W. L. W. 1985, ApJS, 57, 503

Galliano, E., Pantin, E., Alloin, D., \& Lagage, P. O. 2005, MNRAS, 363, L1

Gondoin, P., Lumb, D., Siddiqui, H., Guainazzi, M., \& Schartel, N. 2001, A\&A, 373,805

Ho, L. C., Feigelson, E. D., Townsley, L. K., et al. 2001, ApJ, 549, L51

Hönig, S. F., Beckert, T., Ohnaka, K., \& Weigelt, G. 2006, A\&A, 452, 459

Keel, W. C. 1983, ApJS, 52, 229

Krabbe, A., Böker, T., \& Maiolino, R. 2001, ApJ, 557, 626

Kraemer, S. B., George, I. M., Crenshaw, D. M., \& Gabel, J. R. 2004, ApJ, 607, 794

Lagage, P. O., Pel, J. W., Authier, M., et al. 2004, The Messenger, 117, 12

Landi, R., Bassani, L., Malaguti, G., et al. 2001, A\&A, 379, 46

Lumsden, S. L., Alexander, D. M., \& Hough, J. H. 2004, MNRAS, 348, 1451

Lutz, D., Maiolino, R., Spoon, H. W. W., \& Moorwood, A. F. M. 2004, A\&A, 418,465

Nenkova, M., Ivezic, Z., \& Elitzur, M. 2002, ApJ, 570, L9

Pier, E. A., \& Krolik, J. H. 1993, ApJ, 418, 673

Reeves, J. N., Nandra, K., George, I. M., et al. 2004, ApJ, 602, 648

Reynolds, C. S., Brenneman, L. W., Wilms, J., \& Kaiser, M. E. 2004, MNRAS, 352,205

Rigopoulou, D., Spoon, H. W. W., Genzel, R., et al. 1999, AJ, 118, 2625

Risaliti, G. 2002, A\&A, 386, 379

Risaliti, G., Gilli, R., Maiolino, R., \& Salvati, M. 2000, A\&A, 357, 13

Smith, D. A., \& Done, C. 1996, MNRAS, 280, 355

Spergel, D. N., et al. 2003, ApJS, 148, 175

Steenbrugge, K. C., Kaastra, J. S., Blustin, A. J., et al. 2003, A\&A, 408, 921

Sturm, E., Hasinger, G., Lehmann, I., et al. 2006, ApJ, 642, 81

Terashima, Y., Iyomoto, N., Ho, L. C., \& Ptak, A. F. 2002, ApJS, 139, 1

Turner, T. J., \& Pounds, K. A. 1989, MNRAS, 240, 833

Urry, C. M., \& Padovani, P. 1996, PASP, 107, 803

Véron-Cetty, M.-P., \& Véron, P. 2000, ESO Scientific Report, 19 